BeAle, G. H. \& KACSER, H. (1957). J. gen. Microbiol. 17, 68-74

\title{
Studies on the Antigens of Paramecium aurelia with the Aid of Fluorescent Antibodies
}

\author{
By G. H. BEALE AND H. KACSER \\ Department of Animal Genetics, University of Edinburgh
}

SUMMARY: Preparations of Paramecium aurelia, which had been immobilized by placing the organisms in a solution containing antiserum conjugated with fluorescein (conjugated antiserum), were examined by fluorescence microscopy. Unfixed paramecia accumulated fluorescent antibody in a thin layer around the entire surface of the organisms, and in globules at the clumped tips of the cilia, but not in the cilia themselves. No fluorescence was seen in the nuclei or the cytoplasm, but the food vacuoles became brightly fluorescent when the paramecia remained in conjugated antiserum for a few hours. Paramecia, which had been fixed with osmic acid and subsequently treated with fluorescent antibody, showed a faint fluorescence along the whole lengths of the cilia. When transformation from one serotype to another took place, the change in ability to take up a given kind of fluorescent antibody was seen to occur uniformly over the whole surface of the organism. It is concluded that the immobilization antigen is a fluid substance which covers the whole surface of the paramecia and is exuded into the medium under certain conditions, especially when homologous antibody is present.

Extensive studies have been made on the genetics of antigen variation in Paramecium aurelia by Sonneborn, Beale and others (for references, see Beale, $1954,1957)$. In all such work only one immunological reaction has been used, namely, the immobilization of the organisms which takes place when living paramecia are placed in a fluid containing dilute homologous antiserum. It is known that this immobilization reaction is associated with a clumping of the cilia, and for this reason the antigens concerned have been generally referred to as the 'ciliary antigens'. However, definite evidence that these antigens are derived from part of the ciliary structure has never been brought forward.

The paramecium antigen system, with its complex array of determining factors in the nucleus, cytoplasm and environment, has been considered as a model, valuable for the understanding of mechanisms of cell differentiation generally (Sonneborn, 1947; Beale, 1952). It is therefore important to obtain as much information as possible concerning the nature and location of the final products in the system, namely the specific antigens themselves. The elegant technique developed by Coons and collaborators (Coons, Creech, Jones \& Berliner, 1942; Coons \& Kaplan, 1950), offers a means for localizing the sites of deposition of antibodies in tissues, and thus enables inferences to be drawn regarding the sites of the corresponding antigens. Essentially the method involves conjugating the antibodies in suitable antisera with a fluorochrome such as fluorescein, applying the conjugated antiserum thus obtained to the tissue under investigation, washing away the fluorescent solution, and observing the treated material by fluorescence microscopy, that is, under 
ultraviolet (u.v.) irradiation. The characteristic bright green fluorescence of fluorescein indicates the site of deposition of conjugated antibody. In such tests it is important to set up suitable controls, since the conjugated antiserum contains other conjugated proteins which may be taken up non-specifically by the material under study and give spurious indications of an uptake of fluorescent antibody. Furthermore, exchange of fluorescein from antibody to other material must be eliminated as a possible cause of localized fluorescence. With the antigens of Paramecium, such controls may be conveniently obtained by exposing paramecia of a different serotype to precisely the same treatment as the homologous organisms, as will be described.

In the experiments here reported, whole paramecia were exposed to fluorescent antiserum. Hence, only the external parts of the cell were studied. In future work it is hoped to apply fluorescent antiserum to sectioned material, and to examine whether there are substances related to the immobilization antigens in the interior of the organism, in the cytoplasm and nucleus, where it is known from genetic evidence that the determinants of the immobilization antigens are situated.

\section{METHODS}

A sample of immune rabbit antiserum, developed against Paramecium aurelia stock 168 (variety 1 ), serotype G, was used. Paramecia of serotype 168G were the homologous test objects; and types $168 \mathrm{D}$ and $90 \mathrm{G}$ were the nonhomologous control organisms. Details concerning the preparation of antisera have been described previously (Sonneborn, 1950; Beale, 1954, 1957). Suspensions of broken-up paramecia were used for injections into rabbits. The conjugation of the antiserum with fluorescein was affected by the method of Coons \& Kaplan (1950). Whole serum was used. We are greatly indebted to Dr A. B. Roy for carrying out this part of the work, and for supplying a sample of fluorescein isocyanate.

The conjugated antiserum obtained was diluted about one part in twentyfive with distilled water, and this was the solution used in all tests. Samples were kept frozen, and thawed as required. The comparatively high concentration of conjugated antiserum which had to be used was a consequence of the considerable lowering in titre of the antiserum, as measured by the immobilization reaction (from $1 / 800$ to $1 / 100$ ) due, presumably, to the chemical procedures used in conjugating antibody with fluorescein.

Three methods of preparing the paramecia for examination by fluorescence microscopy were used: (1) live paramecia were simply treated with dilute fluorescent antiserum, washed with dilute buffered Ringer solution, and examined while still living, though immobilized; (2) after treatment and washing as above, paramecia were fixed by exposing them to osmic acid vapour for $30 \mathrm{sec}$. (or by placing them in very dilute buffered osmic acid solution); (3) paramecia were first fixed in osmic acid, then placed in the dilute fluorescent antiserum, and finally washed and examined.

In order to obtain satisfactorily bright fluorescent preparations (i.e. suitable for colour photography), it was found necessary to expose the paramecia very 
thoroughly to the conjugated antiserum, e.g. living paramecia were treated about three times longer than necessary to bring about immobilization, and osmic acid-fixed specimens were left in the fluorescent antiserum (at a dilution of about $1 / 50$ ) overnight.

After treatment, the paramecia were placed on a clean glass slide in a small drop of dilute (1/10) Ringer solution, and a u.v.-absorbing coverslip placed on top, supported at the sides by two thin pieces of coverslip. A 250 W. mercury vapour lamp was used as a source of u.v. radiation, and visible light was excluded by suitable filters. Glass lenses were found to be adequate. Most of the preparations were examined with a dark ground condenser.

\section{RESULTS}

Before describing the effect of fluorescent antiserum on paramecia, we may consider briefly the immobilization process in so far as it can be studied with non-fluorescent material observed under the phase-contrast microscope. As shown in Pl. 1, figs. 1 and 2, when paramecia were immobilized by antiserum, the cilia clumped together at their tips, where there was an accumulation of lumps of 'sticky' substance. Such material may also accumulate in the solution around the paramecia, especially at the posterior ends of the ciliates. Similar observations have been recorded by other workers (e.g. Harrison, 1955), but there has been no proof that these accumulations of 'sticky' material actually contained antigen-antibody complexes. It is possible that bacteria and other debris from the medium contributed a large part of the bulk of the lumps.

The cytoplasm of paramecia which had been immobilized by specific antiserum continued to circulate actively; and even the cilia could be seen to be in a state of agitation, making such irregular contractions and lateral movements as are possible when the tips are stuck together. The special gullet cilia, moreover, were apparently quite unaffected by specific antiserum. Trichocysts could occasionally be seen to be extruded as a consequence of treatment with antiserum, and this was especially pronounced in immobilized paramecia which were subsequently fixed with osmic acid vapour.

When fluorescent antiserum was applied to paramecia of homologous serotype (i.e. type 168G) by method (1) (see above, p. 69), and examined under u.v. radiation, fluorescence appeared in a thin layer around the entire surface of the organism, in globules (waving back and forth) at positions corresponding to the clumped tips of the cilia, and in granules interspersed in the 'sticky' material at the posterior ends of the paramecia. The cilia themselves were not fluorescent, except for the large stationary caudal cilia, which were sometimes faintly green along their length. There was no fluorescence in the gullet. Finally, a few food vacuoles were brightly fluorescent, and could be seen circulating around the cytoplasm. The nuclei and cytoplasm were invisible when examined by fluorescence microscopy.

Non-homologous paramecia of serotypes $90 \mathrm{G}$ or $168 \mathrm{D}$ (i.e. those differing from type $168 \mathrm{G}$ by either genic or cytoplasmic factors, respectively) after 
being placed in the conjugated anti-168G serum, did not fluoresce on the surface at all, and were of course not immobilized. Such paramecia did, however, ingest the fluorescent material, and when allowed to do so for $12 \mathrm{hr}$. or more showed a large number of intensely green fluorescent food vacuoles. It is assumed that the accumulation of fluorescent material in the food vacuoles had nothing to do with specific uptake of antibody by antigen, but was purely mechanical.

The specificity of the uptake of the fluorescent antibody by paramecia could be very clearly demonstrated with the aid of pairs of conjugating animals. In each pair, it was so arranged that one animal was of serotype $168 \mathrm{G}$, while the mate was of some other serotype. It was found to be important to have the pairs firmly joined together before treating them with fluorescent antiserum, and this was achieved by allowing conjugation to go on normally for $2 \mathrm{hr}$. or more at $27^{\circ}$ in ordinary culture fluid. Such pairs exhibited a remarkable differential uptake of fluorescent material: the homologous member (168G) showed a green deposit around the surface, the other did not show the slightest trace of fluorescence on the surface, even in those regions where the cilia of the two mates were extremely close together. Both members of the pair, but particularly the non-homologous one, sometimes accumulated fluorescent material in some of the food vacuoles.

It might be argued that paramecia which have been treated with homologous antiserum and are therefore in a 'sticky' condition, would be liable to take up non-specifically any material present in the medium, and this would include, in these experiments, fluorescent substances. To check this point, the experiment described in the previous paragraph was also performed in a modified form, as follows. Pairs of conjugating paramecia (of diverse serotype) were treated first with a mixture of ordinary, non-fluorescent antisera capable of immobilizing both members of a given pair, and subsequently with fluorescent antibody effective against only one of them. Here also it was found that only one member of each pair became fluorescent, even though the other member had been immobilized, and was presumably in a 'sticky' condition.

All observations made on living paramecia, prepared according to method (1) had to be made quickly, since the intense beam of u.v. radiation kills the organism in a few seconds. Amongst effects observed during this killing by u.v.-irradiation was a movement of green fluorescent material away from the paramecium into the solution.

After preparation by method (2), i.e. with final fixation in osmic acid, the paramecia showed essentially the same distribution of fluorescence as in the living material described above, but the differentiation between fluorescent and non-fluorescent regions was not quite as sharp, partly due to the fact that in fixed material the protoplasm itself showed a faint bluish fluorescence. Fixed material had the advantage, however, of remaining unchanged after exposure to u.v. radiation for some time, though eventually the green colour was bleached out. As mentioned above, trichocysts were liable to be thrown out in quantity when paramecia were immobilized by antiserum and fixed 
with osmic acid. When this happened, the mass of extruded trichocysts brought out with it quantities of green fluorescent granules similar to those which collect at the tips of the cilia. The extruded trichocysts themselves were sometimes fluorescent, sometimes not. It is possible that trichocysts which are extruded during the period of exposure to conjugated antiserum become coated with fluorescent material, while those which are brought out by the final fixation are non-fluorescent.

Paramecia which were prepared by method (3), i.e. fixed with osmic acid before being treated with conjugated antiserum, showed an appearance somewhat different from that described above. The cilia of organisms treated according to method (3) did not clump at their tips, and there were no fluorescent granules either at the tips of the cilia or adhering to the exterior of the organism. What was very distinctive here was that the cilia were clearly fluorescent along their entire lengths. The pellicle was, as before, fluorescent around the whole surface. Finally, there were of course no fluorescent food vacuoles since the paramecia were dead before being put into the diluted conjugated antiserum.

It should be emphasized that the uptake of fluorescent antibody by cilia which had been previously fixed with osmic acid was highly specific, as shown by the failure of antigenically non-homologous paramecia, treated in precisely the same way, to show any such uptake.

After finding that fixation with osmic acid did not destroy the ability of paramecia to take up homologous antibody specifically, a number of other treatments were tried, such as fixation with formaldehyde and heating to $70^{\circ}$ for 2-3 min. Such treatments resulted in the conversion of the paramecia to a form in which all of them, whether antigenically homologous or not, acquired a fluorescent coating. It is presumed that this non-specific uptake had nothing to do with absorption of specific antibodies by antigens on the surface of the paramecia, for it should be remembered that the diluted fluorescent antiserum contained other proteins conjugated with fluorescein, in addition to the immobilizing antibodies.

Observations were made on the distribution of antigen in paramecia which were in the process of transforming from one serotype to another. The process by which paramecia transform, under the influence of various environmental treatments, has been described elsewhere in detail (Beale, 1954, 1957). Paramecia of serotype $168 \mathrm{G}$ can be very easily caused to give rise to organisms of serotype $168 \mathrm{D}$ by growth at a high temperature $\left(27^{\circ}-35^{\circ}\right)$. In the present studies, some organisms of type $168 \mathrm{G}$ were separately isolated into culture fluid and incubated at $35^{\circ}$ for various periods up to $12 \mathrm{hr}$., by which time the transformation to $168 \mathrm{D}$ was practically complete. At intervals during the transformation process samples were taken, fixed with osmic acid, treated with fluorescent antiserum, washed and examined. It was found that as the reaction of the organisms to anti- $168 \mathrm{G}$ serum diminished, so did their ability to take up specific fluorescent antibody. The diminution in ability to take up the specific fluorescent antibody was seen to be a gradual process, though it was not possible to decide whether the cilia, or certain regions of them, lost 
the ability to take up the fluorescent antibody at an earlier stage than the pellicle, because the fluorescence of the cilia was at all times faint. What, however, was clear was that the change in the antigen occurred uniformly all around the organism and did not occur only at regions where new cilia and pellicle were being developed. In this connexion it is worth recalling that during fission there is thought to be an abundant production of new cilia in a band approximately around the equator of the animal (Wichterman, 1953), and fission occurred during transformation.

\section{CONCLUSIONS}

From the observations reported in this paper, it is concluded that the antigen concerned with the immobilization reaction in paramecia is found on the pellicle and on the cilia, but may diffuse into the medium under the influence of certain treatments. Fixation with osmic acid does not destroy the antigen, but prevents it from diffusing away from the site at which it is formed, possibly by making the external membrane impermeable to the antigen, though not, apparently, to antibody. From the fact that during transformation, in actively growing organisms, from one serotype to another, there is a uniform change in the antigen all over the surface, it is deduced that the antigen is not associated with any particular structure, such as the cilia. Rather would it seem that the antigen is a fluid substance which can cover any part of the exterior surface of the paramecium. When homologous antibody is added, it is thought that there is an exudation of antigen, which unites with the antibody and forms the 'sticky' lumps at the tips of the cilia and elsewhere. The precise cause for this accumulation is not clear, but it may be mechanical. The antigen-antibody product never sticks to the cilia of nonhomologous animals, however close they may be.

Views somewhat similar to the above were put forward by Harrison (1955), arising out of observations, with phase-contrast microscopy, on the immobilization reaction in Paramecium, Tetrahymena and Colpoda. This author considered that the 'slimy accumulation' formed around ciliates when homologous antibody is added, is an antigen-antibody product, since it appeared in any and all combinations of strains of ciliates and antisera which gave precipitative and complement-fixation reactions, but in no other situations. Harrison also noted a loss in volume of paramecia during immobilization, and assumed this to be due to the exudation of the antigen. Such a pouring out of exudate, which passes out into the surrounding medium and combines with antiserum, was described by Robertson (1939), with Tetrahymena. Amongst other observations recorded by her was one to the effect that the cilia were not attacked by the antiserum, but were withdrawn from the antigen-antibody 'sheath', and could later resume normal activity.

In view of the diffusible properties of the paramecium antigen it seems likely that the latter is constantly being exuded into the water in which the paramecia are swimming, but we have no definite proof that this is so. It is, however, known that a soluble antigen can be readily extracted from the paramecia (Finger, 1957; Van Wagtendonk, van Tijn, Litman, Reisner \& 
Young, 1956) and that this soluble antigen specifically lowers the titre of the antibodies concerned with the immobilization reaction.

As a result of the conclusions arrived at in this paper, we feel that the term 'ciliary antigen' is inappropriate for a substance which is found, not only on (or in) the cilia, but also, and most abundantly, on the pellicle, and which may well be continuously passing into the medium. 'Immobilization antigen' would in our opinion be a better term.

We wish to thank Drs A. B. Roy and R. Alexander for much help in the preparation of the fluorescent antiserum and for technical advice generally.

\section{REFERENCES}

Beale, G. H. (1952). Antigen variation in Paramecium aurelia, variety 1. Genetics, $37,62$.

Beale, G. H. (1954). The Genetics of Paramecium aurelia. Cambridge University Press.

Beale, G. H. (1957). The antigen system of Paramecium aurelia. Int. Rev. Cytol. 6, 1.

Coons, A. H., Creech, H. J., Jones, R. N. \& Berliner, E. (1942). The demonstration of pneumococcal antigen in tissues by the use of fluorescent antibody. J. Immunol. 45, 159.

Coons, A. H. \& Kaplan, M. H. (1950). Localization of antigen in tissue cells. II. Improvements in a method for the detection of antigen by means of fluorescent antibody. J. exp. Med. 91, 1.

Finger, I. (1957). Immunological studies of the immobilization antigens of Paramecium aurelia variety 2. J. gen. Microbiol. 16, 350.

Harrison, J. A. (1955). General aspects of immunological reactions. Chap. 7 in Biological Specificity and Growth (ed. E. G. Butler). Princeton University Press.

Robertson, M. (1939). A study of the reactions in vitro of certain ciliates belonging to the Glaucoma-Colpidium group to antibodies in the sera of rabbits immunized therewith. J. Path. Bact. 48, 305.

Sonneborn, T. M. (1947). Developmental mechanisms in Paramecium. Growth 11, 291.

SonNebonn, T. M. (1950). Methods in the general biology and genetics of Paramecium aurelia. J. exp. Zool. 113, 87.

Wagtendonk, W. J. Van, TiJn, B. Van, Litman, R., Reisner, A. \& Young, M. L. (1956). The surface antigens of Paramecium aurelia. J. gen. Microbiol. 15, 617.

Wichterman, R. (1953). The Biology of Paramecium. New York: The Blakiston Co.

\section{EXPLANATION OF PLATE}

Fig. 1. Unimmobilized paramecium, fixed in osmic acid vapour, photographed in phasecontrast microscope. $\times 500$.

Fig. 2. Paramecium immobilized with homologous antiserum, then fixed and photographed as Fig. 1. $\times 500$. 
Journal of General Microbiology, Vol. 17, No. 1

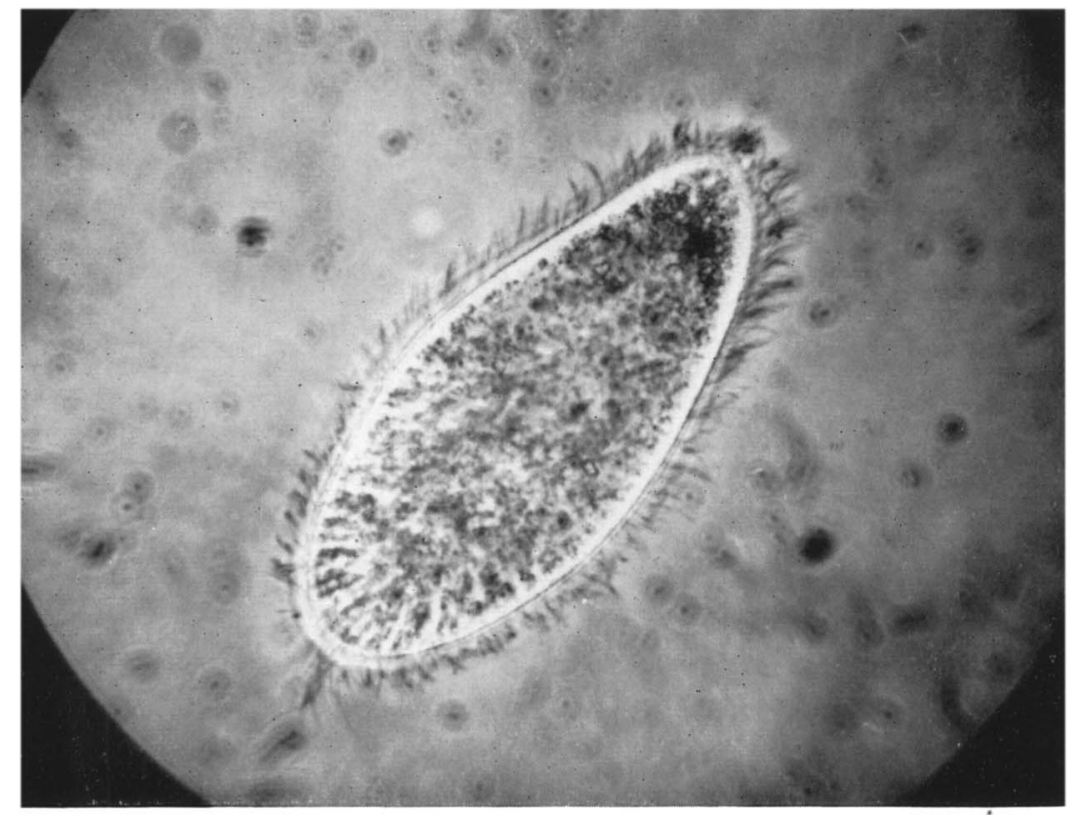

Fig. 1

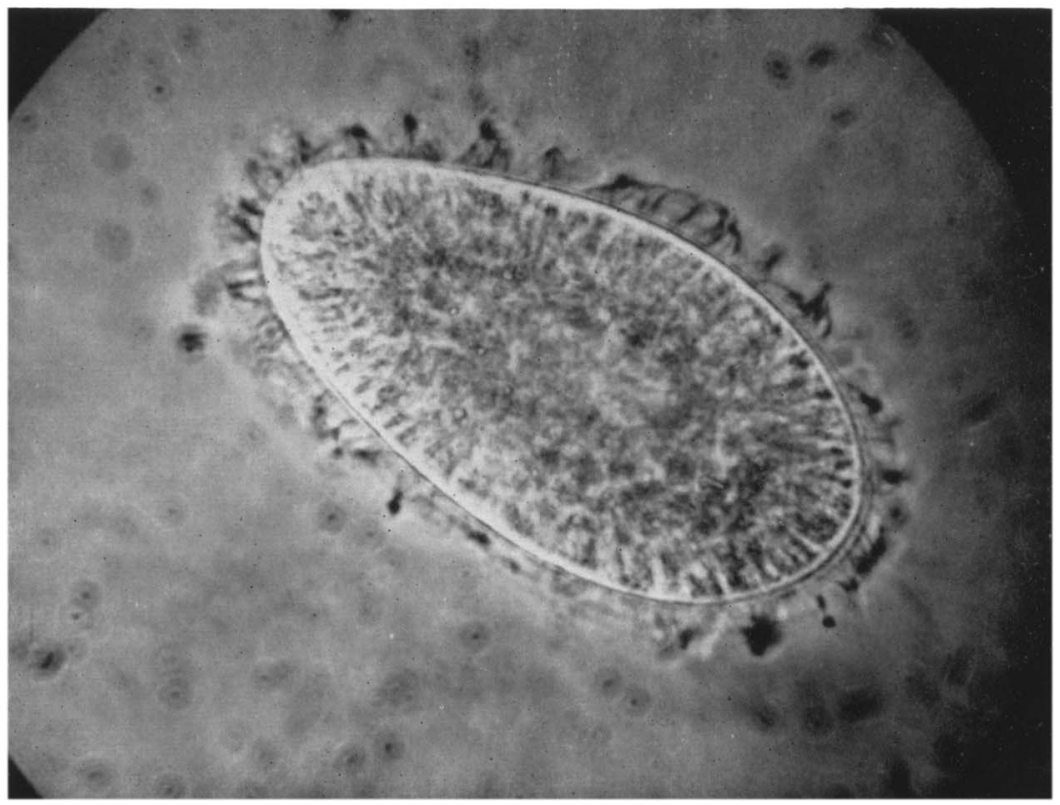

Fig. 2

G. H. Beale and H. Kacser-Antigens of Paramecium aurelia. Plate 1 (Facing $p$. 74) 